\title{
Electromechanical Dynamics For Microplate
}

\author{
Lizhong Xu and Zhentong Wu \\ Mechanical engineering institute, Yanshan University, Qinhuangdao 066004, China
}

\begin{abstract}
(Received 5 November 2007; accepted 21 January 2009)
In this paper, an electromechanical coupled dynamic model of the microplate subjected to electrostatic force is presented. The dynamic equations and the static equations of the electromechanical coupled microplate are obtained. For three different boundary conditions, the dynamic equations and the static equations are resolved, and the natural frequencies and the vibrating modes of the microplate subjected to electrostatic force are investigated. Influences of the boundary conditions on the natural frequencies and the vibrating modes are analyzed. Influences of the main electromechanical parameters on the natural frequencies are investigated as well. The natural frequencies of the microplate subjected to electrostatic force are affected by mechanical and electric parameters such as microplate size, voltage, clearance, etc.
\end{abstract}

\section{INTRODUCTION}

Microelectromechanical systems (MEMS) are integrated systems consisting of microelectronics, microactuators and, in most cases, microsensors. For more than a decade, research on MEMS has been conducted by almost all major universities and research institutions. ${ }^{1-3}$

A typical MEMS structures consist of thin beams with crosssections in the order of microns $(\mu m)$ and lengths in the order of ten to hundreds of microns. Sometimes, MEMS structural elements are thin plates or thin rings. An example is a small rectangular silicon plate with sides in the order of milimeters and thickness of the order of microns, that deforms when subjected to electric fields. Owing to its small size, significant forces and deformations can be obtained with the application of low voltages. Examples of devices that utilize vibrations of such plates are synthetic microjets, and microspeakers, etc. ${ }^{4}$ Another example is micromechanical gyroscopes which are exclusively of vibrating types, including double-gimbals structure, and cantilever beam structure, tuning-fork structure and vibrating ring structure. ${ }^{5-8}$

Understanding the dynamic behavior of MEMS is very important for developing new MEMS devices and controlling their performance. Electrostatic actuation is the most frequently applied principle combing versatility and simple technology. Then, the focus of the microsystem dynamics is the electromechanical coupled dynamics of the micro beam and microplate under electric field.

To date, many academic investigations on solving dynamic electromechanical coupling problems for MEMS structures have been reported. McCarthy et al. applied the finite difference methods to analyze the switching period and transient response of MEMS switches fabricated using electrodeposition. ${ }^{9}$ Kuang and Chen utilized the differential quadrature method to evaluate the spring softening effect and to predict the natural frequency of a fixed-fixed beam subjected to electrostatic loading ${ }^{10-13}$ and the results agree with the experimental data in large extent. ${ }^{14}$ Most of the commercial MEMS design packages, such as MEMS Pro, as well as some general purpose finite element packages such as ANSYS, have been used to solve electromechanical coupled dynamics problems. ${ }^{4,15}$
Sometimes, the analytical solution has obvious advantage over numerical solution. The analytical solution can give inherent law in the electromechanical coupled dynamics system obviously. It is very favorable to dynamic parameter design and optimum of the MEMS system. However, obtaining analytical solution of the problem is difficult. The easiest analytical model describing the dynamics behavior of the MEMS system is the one-dimensional (1-D) lumped approach which has been extensively used to model electrostatic actuators. ${ }^{16-20}$ Using this type of the models, complex non-linear dynamics behaviors - such as shifting of the natural frequencies, bifurcation and chaos caused by the non-linear electrostatic forces in MEMS devices with variable capacitors - have been investigated as well. ${ }^{21-24}$

In these 1-D models, the vibrating modes of the micro beam or microplate are not considered. Hence, some models about micro beam with distributed parameters were presented, and the vibration was described by boundary-value problems, which consist of partial differential equations under given boundary conditions. ${ }^{25-27}$ Lizhong Xu proposed an electromechanical coupled dynamic model of the micro beam with distributed parameters and investigated its electromechanical coupled dynamic behavior. ${ }^{25}$ Abdel-Rahman et al. presented a non-linear model involving an electrostatic force of an air gap capacitor, a restoring force of the microbeam as well as an axial load applied, and subsequently, the eigenvalue problem, describing the vibration of the microbeam around its static equilibrium deflected position, is solved numerically to establish natural frequencies and mode shapes. ${ }^{26,27}$

The electromechanical coupled dynamics of the microplate considering its vibrating modes has not been developed yet. It is a harder problem in which partial deferential equation including two position variables should be resolved. In electromechanical coupled dynamics model of the micro beam with distributed parameters, the partial deferential equation only including one position variables was resolved.

In this paper, an electromechanical coupled dynamics model of the microplate with distributed parameters subjected to electrostatic force is presented and its electromechanical coupled dynamic equation is given. The dynamic equation is divided into a linear dynamic equation for dynamic displacement and 